$02.1 ; 01.5$

\title{
Динамика локализованной кольцевой нелинейной волны в углеродной нанотрубке
}

\author{
(C) О.Е. Глухова ${ }^{1,2}$, А.П. Четвериков ${ }^{1}$, В.В. Шунаев ${ }^{1, \uparrow}$ \\ ${ }^{1}$ Институт фризики, Саратовский национальный исследовательский государственный университет \\ им. Н.Г. Чернышевского, Саратов, Россия \\ ${ }^{2}$ Институт бионических технологий и инжиниринга, Первый Московский государственный медицинский университет \\ им. И.М. Сеченова, Москва, Россия \\ ๑ E-mail: vshunaev@list.ru
}

Поступило в Редакцию 1 июня 2021 г.

В окончательной редакции 20 июня 2021 г.

Принято к публикации 21 июня 2021 г.

\begin{abstract}
Методом молекулярно-динамического моделирования исследуется распространение нелинейных локализованных солитоноподобных сверхзвуковых продольных волн в углеродной нанотрубке. Кольцевые волны возбуждаются за счет импульсного воздействия на все атомы торцевого зигзагообразного слоя, в результате чего они приобретают начальную скорость вдоль оси нанотрубки. Показано, что скорость локализованной (солитоноподобной) кольцевой сверхзвуковой волны растет при увеличении начальной скорости торцевых атомов. Приведена рассчитанная зависимость скорости распространяющейся волны от начальной скорости торцевых атомов.
\end{abstract}

Ключевые слова: углеродная нанотрубка, солитон, сверхзвуковая волна.

DOI: 10.21883/PJTF.2021.19.51506.18895

Возмущения с высокой энергией распространяются в двумерных решеточных молекулярных структурах, как правило, в виде локализованных волн (солитонов, бризеров, кинков, краудионов и др.) [1]. Причиной такой динамики является конкуренция нелинейных и дисперсионных свойств решеток, причем первые связаны с нелинейностью потенциалов связи элементов решеток, а вторые обусловлены дискретностью решеточных структур. Распространение волн вдоль какой-то кристаллографической оси происходит естественным образом, если вдоль этой оси элементы решетки распределены эквидистантно, как, например, в треугольной решетке [2-5]. При этом волна может распространяться или только в одном ряду решетки или в $M$ смежных рядах [3]. Однако волна имеет ограниченное время жизни, поскольку часть энергии тратится на возмущение колебаний в смежных рядах. Время жизни и длина траектории волны до ее разрушения растут при увеличении числа $M$ первоначально возбужденных рядов, поскольку энергию теряют в основном бегущие возмущения во внешних рядах, связанных с невозмущенной сначала частью решетки. Например, в треугольной решетке с потенциалом Морзе эта зависимость почти линейная [5]. Но рассеяние энергии в невозмущенные ряды гораздо быстрее происходит в решетках, в которых нет направлений, вдоль которых атомы были бы расположены равномерно, как, например, в гексагональной решетке графена (см., например, работу [6] и обширную библиографию, приведенную в ней). Однако эффект перекачки энергии в первоначально невозмущенные ряды естественным образом не реализуется, если двумерная решетка свернута в трубку, а начальное возмущение скорости вдоль оси трубки прикладывается ко всем торцевым атомам, в результате чего в трубке возникает кольцевая волна.

В настоящей работе объектом исследования является углеродная нанотрубка $(16,0)$, поскольку именно такого типа трубки являются наиболее часто синтезируемыми среди ахиральных [7]. Рассматривается динамика распространения локализованной солитоноподобной кольцевой волны, возникающей при импульсном возбуждении продольной скорости у каждого концевого атома зигзагообразного торца перпендикулярно обрезанной трубки. Волна бежит вдоль направления „кресло“, причем возбуждения в каждом продольном ряду атомов идентичны, что обеспечивается ахиральностью трубки. Основной целью работы является определение скорости солитоноподобной волны как функции начального импульса (начальной скорости), получаемого торцевыми атомами.

Эволюция смещения и скорости каждого атома трубки исследуется в ходе проведения молекулярнодинамического исследования на основе метода сильной связи в приближении теории функционала плотности SCC DFTB (self-consistent charge density functional tight-binding) [8], согласно которому полная энергия системы определяется выражением

$$
E_{t o t}=E_{\mathrm{BS}}+E_{r e p}+E_{\mathrm{SCC}}
$$

где $E_{\mathrm{BS}}-$ энергия зонной структуры, $E_{r e p}-$ энергия отталкивания, $E_{\mathrm{SCC}}$ - поправка, учитывающая самосогласование зарядов на атомах. Данный метод позволяет учитывать, в частности, смещения электронных облаков каждого атома, что делает численный эксперимент максимально приближенным к реальному. Ранее метод 

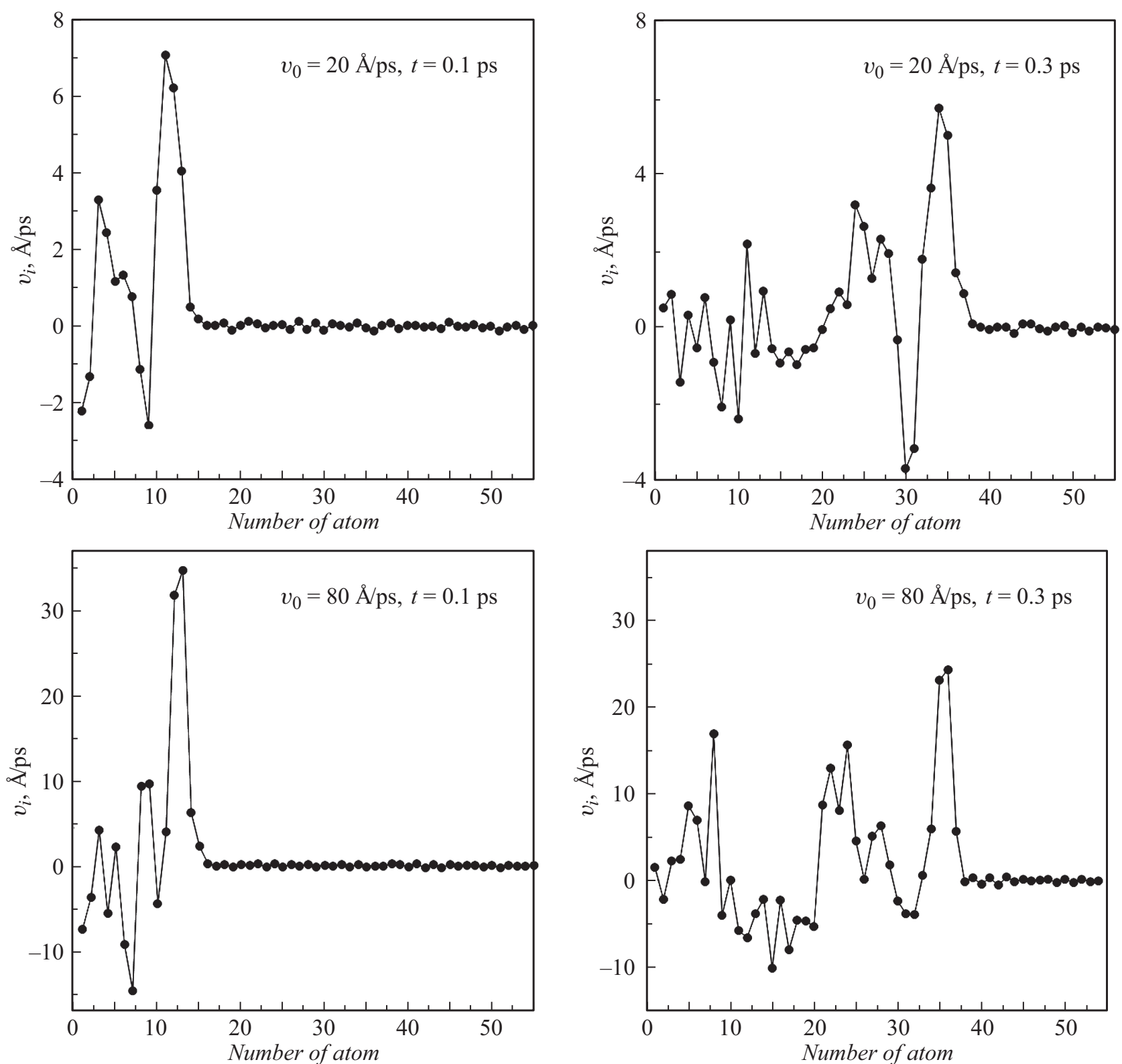

Рис. 1. Распределение продольной скорости атомов $v_{i}$ в одном продольном ряду нанотрубки в последовательные моменты времени $t=0.1$ и 0.3 ps при небольшой $\left(v_{0}=20 \AA / \mathrm{ps}\right)$ и высокой $\left(v_{0}=80 \AA / \mathrm{ps}\right)$ начальной скорости торцевых атомов.

SCC DFTB был успешно применен для моделирования роста углеродных нанотрубок [9], а также анализа их механических и электронных свойств [10]. На рис. 1 показаны распределения скорости атомов в одном из рядов трубки, ориентированном вдоль ее оси, в последовательные моменты времени в случае небольшого начального импульсного воздействия (верхние фрагменты) и в случае существенного воздействия (нижние фрагменты).

Из представленных рисунков следует, что распространяющееся вдоль трубки локализованное возмущение может быть классифицировано как сверхзвуковая солитоноподобная волна, поскольку оно слабо деформируется при движении и является сверхзвуковым, так как никакие возмущения впереди него не наблюдаются. Однако динамические возмущения структуры трубки после прохождения начального импульса в этих экспериментах довольно сильные. Это связано с тем, что начальная форма возмущения в трубке, вызываемая внешним импульсом, не полностью соответствует структуре солитона. Лишь после некоторого переходного процесса выделяется мощный импульс, который бежит со скоростью выше скорости звука, которая по оценкам близка в данном случае к 200-220 А/ps. Длительность переходного процесса равна примерно $0.1 \mathrm{ps}$, что следует из зависимости максимальной по ансамблю скорости частиц от времени (рис. 2). Видно, что к этому времени устанавливается максимальное значение скорости 


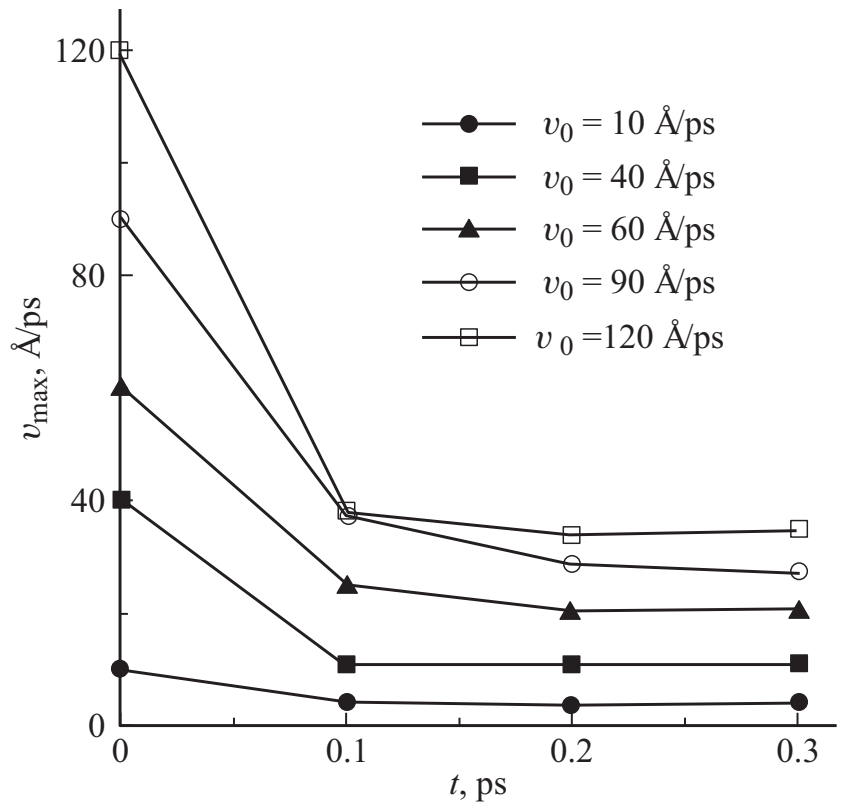

Рис. 2. Эволюция максимальной скорости частиц в нанотрубке при различной начальной скорости частиц торцевого слоя. Видно, что к моменту времени $t=0.1 \mathrm{ps}$ значение максимальной скорости выходит на почти постоянный уровень, что свидетельствует о завершении формирования солитона. В дальнейшем солитон распространяется с почти постоянной скоростью.

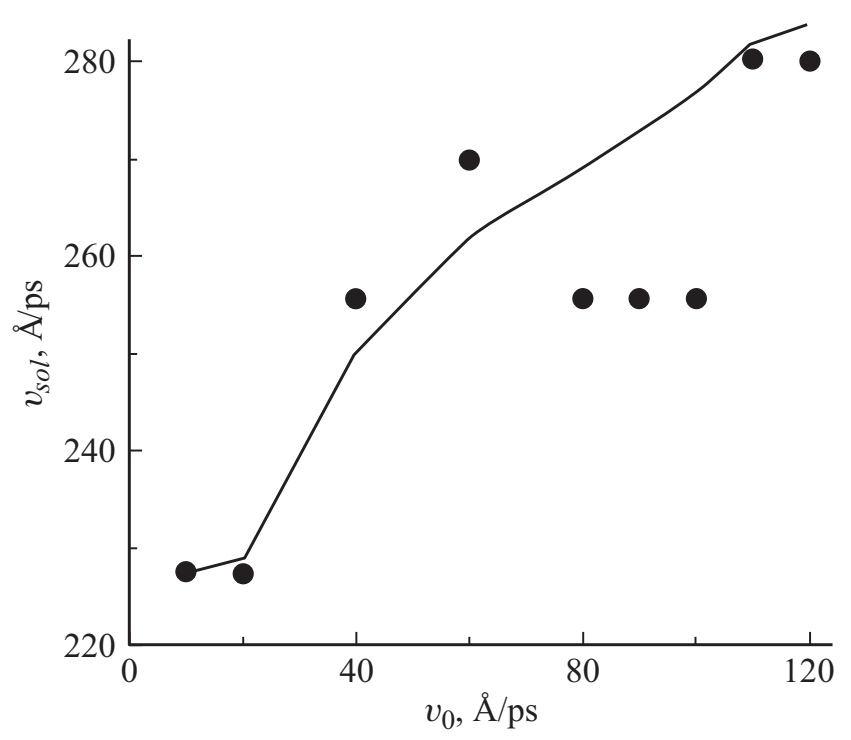

Рис. 3. Зависимость скорости кольцевого солитона в нанотрубке от величины скорости частиц торцевого слоя в начальный момент времени. Точками показаны значения скорости, рассчитанные на интервале времени $0.1-0.2 \mathrm{ps}$, сплошной линией показана усредненная зависимость скорости солитона при различных значениях начальной скорости.

частиц, которое потом почти не изменяется по крайней мере в течение 0.2 ps. Отметим, что длина моделируемой нанотрубки была небольшой - 55 гексагонов (119.7 А).
Установившееся максимальное значение скорости частиц в сформировавшемся бегущем импульсе примерно в 3 раза меньше начального значения $v_{0}$ в диапазоне от 10 до $120 \AA / p s$.

Все указанные значения скорости существенно меньше скорости звука и тем более скорости солитоноподобной волны, определенной на основе результатов компьютерного эксперимента. Ее скорость оценивалась на основе среднего расстояния, пройденного уединенной волной на интервале времени $0.1-0.2 \mathrm{ps,} \mathrm{при} \mathrm{этом}$ координата „солитона“ ассоциировалась с максимумом в распределении скорости частиц (рис. 2). Поскольку это распределение является дискретной функцией, точность определения скорости была не очень высокой из-за того, что пройденное за время $0.1 \mathrm{ps}$ расстояние составляло не более $28.4 \AA$, а шаг дискретизации составлял один гексагон. Тем не менее однозначно показано, что скорость солитоноподобной волны растет с увеличением начальной скорости частиц торцевого слоя (рис. 3). При умеренных значениях $v_{0}$ скорость уединенной волны растет почти пропорционально $v_{0}$, но при значениях, близких к предельным величинам для нанотрубки, не приводящим к ее деформации, зависимость $v_{s o l}\left(v_{0}\right)$ демонстрирует стремление к насыщению при значении $\sim(1.4-1.5)$ звуковой скорости (если принять, что скорость звука в углеродной нанотрубке составляет в условиях эксперимента $\sim 200 \AA / \mathrm{ps}$ ). С учетом того, что энергия начального возбуждения растет пропорционально $v_{0}^{2}$, можно заключить, что такое повышение скорости волны примерно на 40-50\% происходит при увеличении начальной энергии более чем в 100 раз.

При распространении волны атомы трубки, по-видимому, совершают движения не только вдоль ее поверхности, но и поперек поверхности, на что также тратится энергия волны. Однако этот вопрос требует отдельного рассмотрения. Также одним из возможных направлений продолжения исследований является рассмотрение солитоноподобных волн в неуглеродных нанотрубках [11].

\section{Финансирование работы}

А.П. Четвериков (постановка задачи, обсуждение результатов моделирования и их описание) выражает благодарность за финансовую поддержку Российскому фонду фундаментальных исследований в рамках совместного с DFG научного проекта № 20-52-12004. Работа В.В. Шунаева (проведение расчетов, оформление статьи) выполнена при поддержке стипендии Президента РФ молодым ученым и аспирантам (проект № СП-3976.2021.1).

\section{Конфликт интересов}

Авторы заявляют, что у них нет конфликта интересов. 


\section{Список литературы}

[1] Е.А. Корзникова, С.Ю. Фомин, С.В. Дмитриев, Локализованные колебания в двумерных решетках (Наукоемкие технологии, СПб., 2018).

[2] P.G. Kevrekidis, B.A. Malomed, Yu.B. Gaididei, Phys. Rev. E, 66, 016609 (2002). DOI: 10.1103/PhysRevE.66.016609

[3] A.P. Chetverikov, W. Ebeling, M.G. Velarde, Physica D, 240, 1954 (2011). DOI: 10.1016/j.physd.2011.09.005

[4] С.В. Дмитриев, Е.А. Корзникова, А.П. Четвериков, ЖЭТФ, 153 (3), 417 (2018).

[5] A.P. Chetverikov, S.V. Dmitriev, W. Ebeling, E.A. Korznikova, M.G. Velarde, Mater. Phys. Mech., 35(1), 16 (2018). DOI: $10.18720 / M P M .3512018 \_3$

[6] I.A. Shepelev, A.P. Chetverikov, S.V. Dmitriev, E.A. Korznikova, Comput. Mater. Sci., 177, 109549 (2020). DOI: 10.1016/j.commatsci.2020.109549

[7] X. Zhao, S. Zhang, Z. Zhu, J. Zhang, F. Wei, Y. Li, MRS Bull., 42 (11), 809 (2017). DOI: 10.1557/mrs.2017.240

[8] M. Elstner, D. Porezag, G. Jungnickel, J. Elsner, M. Haugk, T. Frauenheim, S. Suhai, G. Seifert, Phys. Rev. B, 58 (11), 7260 (1998). DOI: 10.1103/PhysRevB.58.7260

[9] Y. Ohta, Y. Okamoto, S. Irle, K. Morokuma, ACS Nano, 2 (7), 1437 (2008). DOI: $10.1021 / \mathrm{nn} 8001906$

[10] V.V. Ivanovskaya, N. Ranjan, T. Heine, G. Merino, G. Seifert, Small, 1 (4), 399 (2005). DOI: 10.1002/smll.200400110

[11] A. Kochaev, K. Katin, M. Maslov, Comput. Condens. Matter, 16, e00350 (2018). DOI: 10.1016/j.cocom.2018.e00350 